\title{
A Case Control Study of HPV and Abnormal Cervical Cytology Among HIV Infected and Non Infected Females
}

\section{Kavita Karmacharya', Rajiv B Batra ${ }^{2}$ and Subramaniam Kandasamy ${ }^{3}$}

1: Department of Pathology, Nepalese Army Institute of Health Sciences, Shree Birendra Hospital, Chhauni, Kathmandu, Nepal.

2: Department of Pathology, Armed Forces Medical College, Pune, India.

3: G Kuppuswamy Memorial Hospital,Coimbatore, India.

\begin{abstract}
Introduction: HPV is a known cause of abnormal cervical cytology and cervical cancer. The prevalence of cervical cancer among HIV infected women is higher. This study compared the prevalence of HPV and abnormal cervical cytology between HIV positive and negative females.

Methods: In this case-control study, 100 consecutive HIV-positive females were included as cases and 75 HIV-negative females were taken as controls. Cervical smears were taken for Pap smear and HPV by PCR and compared.

Results: Prevalence of HPV among HIV-positive females (35\%; $n=35)$ was higher than HIV-negatives $(8 \% ; n=6)$ and was statistically significant $(\mathrm{P}<0.05)$. The incidence of LSIL, HSIL and ASCUS in the HIV-positive was $16 \%, 3 \%, 2 \%$ respectively and $5 \%, 2.5 \%, 1.3 \%$ respectively in the HIV negative women. LSIL lesion showed statistical significance $(\mathrm{p}<0.05)$ but HSIL and ASCUS lesions had no such significance.

Conclusion: A strong correlation was found between HPV positivity and abnormal cervical lesions diagnosed on cytology, especially among the HIV positive population LSILs. Therefore, screening of cervical cancer should include HPV identification cervical cytology, especially among HIV positive women, so that prompt and appropriate treatment of HIV would help in reducing the prevalence of cervical cancer.
\end{abstract}

Keywords: cervical abnormalities; HIV; human papillomavirus; Pap smear; PCR

Correspondence: Kavita Karmacharya, Department of Pathology, Nepalese Army Institute of Health Sciences, Shree Birendra Hospital Email: kavita_karmacharya@hotmail.com

To cite this article: Karmacharya K, Batra RB, Kandasamy S. A Case Control Study of HPV and Abnormal Cervical Cytology Among HIV Infected and Non Infected Females. Medical Journal of Shree Birendra Hospital. 2017;17(2):3-9.

DOI: http://dx.doi.org10.3126/mjsbh.v16i2.18618

\section{Conflict of Interest: None declared}




\section{INTRODUCTION}

Cervical cancer is one of the most common cancers among the females. For development of cervical cancer, Human papillomavirus (HPV) is a wellknown, important etiologic agent. It works in tandem with several known risk factors - early age at first intercourse, history of multiple sexual partners, oral contraceptive use, high parity, lower socioeconomic status, and immunosuppression. ${ }^{1}$ Immunocompromised women may have a higher risk for persistent HPV infection since the natural history of HPV is altered in them. ${ }^{2,3}$ Thus, HIV positive women with severe immunosuppression have a higher risk of developing cervical cytological abnormalities like squamous intraepithelial lesions (SILs), as compared to HIV negative women. The current study was thus conducted to compare the prevalence of HPV and abnormal cervical cytology among HIV positive and negative females in a tertiary care centre, Pune, India.

\section{METHODS}

A total of 100 consecutively diagnosed HIV-positive females attending Anti-Retroviral Therapy clinic in a tertiary care centre were included in the study. Any patient in the paediatric age group, ones who have undergone hysterectomy, critically ill patients and known cases of cervical carcinoma were excluded. The control group comprised of 75 HIV-negative females attending the Antenatal Health Care Clinic. Informed consent was taken from all the subjects. The study was conducted in a tertiary care centre in Pune, India, for two years during august 2014 and July 2016.

Cervical smears and scrapings were collected from all 175 women and Papanicolaou smears were prepared. The cytological findings were noted and tabulated. Polymerase Chain Reaction (PCR) for HPV 16/18 was performed on the collected scrapings. This was followed by DNA amplification in a thermocycler. Visualisation was done on $2.0 \%$ agarose gel along with a molecular marker. The product size for high risk HPV 16 at 238 bp and HPV 18 at 268 bp was studied. The cervical smear and HPV-Pap findings were tabulated in the masterchart. Epi Info 3.5.1 software was used for statistical analysis and $\mathrm{p}$ value was calculated by Chi square test. P-value $<0.05$ was considered as statistically significant.

Table 1:The baseline age group distribution between the cases and the control groups

\begin{tabular}{|l|c|c|}
\hline Age groups in Years & Cases & Control \\
\hline Less than 20 & 1 & 0 \\
\hline $\mathbf{2 0 - 3 0}$ & 26 & 26 \\
\hline $\mathbf{3 1 - 4 0}$ & 58 & 44 \\
\hline $\mathbf{4 1 - 5 0}$ & 14 & 4 \\
\hline More than 50 & 1 & 1 \\
\hline Mean & 33.7 & 32.29 \\
\hline
\end{tabular}

Table 2: The prevalence of HR-HPV 16/18 among the cases and the controls

\begin{tabular}{|c|c|c|c|}
\hline & $\begin{array}{l}\text { HIV Positive } \\
\text { (N-100) }\end{array}$ & $\begin{array}{c}\text { HIV Negative } \\
\text { (N-75) }\end{array}$ & $\begin{array}{c}\text { p } \\
\text { Value }\end{array}$ \\
\hline $\begin{array}{l}\text { HR-HPV } \\
16 / 18+\end{array}$ & $35(35 \%)$ & $6(8 \%)$ & \multirow{2}{*}{$<0.05$} \\
\hline $\begin{array}{l}\text { HR-HPV } \\
\text { 16/18 - }\end{array}$ & $65(65 \%)$ & $69(92 \%)$ & \\
\hline
\end{tabular}

Table 3:The prevalence of cytological abnormalities among the cases and the controls

\begin{tabular}{|c|c|c|c|}
\hline & $\begin{array}{l}\text { HIV Positive } \\
\text { (N-100) }\end{array}$ & $\begin{array}{l}\text { HIV Negative } \\
\text { (N-75) }\end{array}$ & P Value \\
\hline LSIL & $16(16 \%)$ & $4(5 \%)$ & 0.011 \\
\hline HSIL & $3(3 \%)$ & $2(2.5 \%)$ & 0.65 \\
\hline ASCUS & $2(2 \%)$ & $1(1.3 \%)$ & 0.56 \\
\hline
\end{tabular}



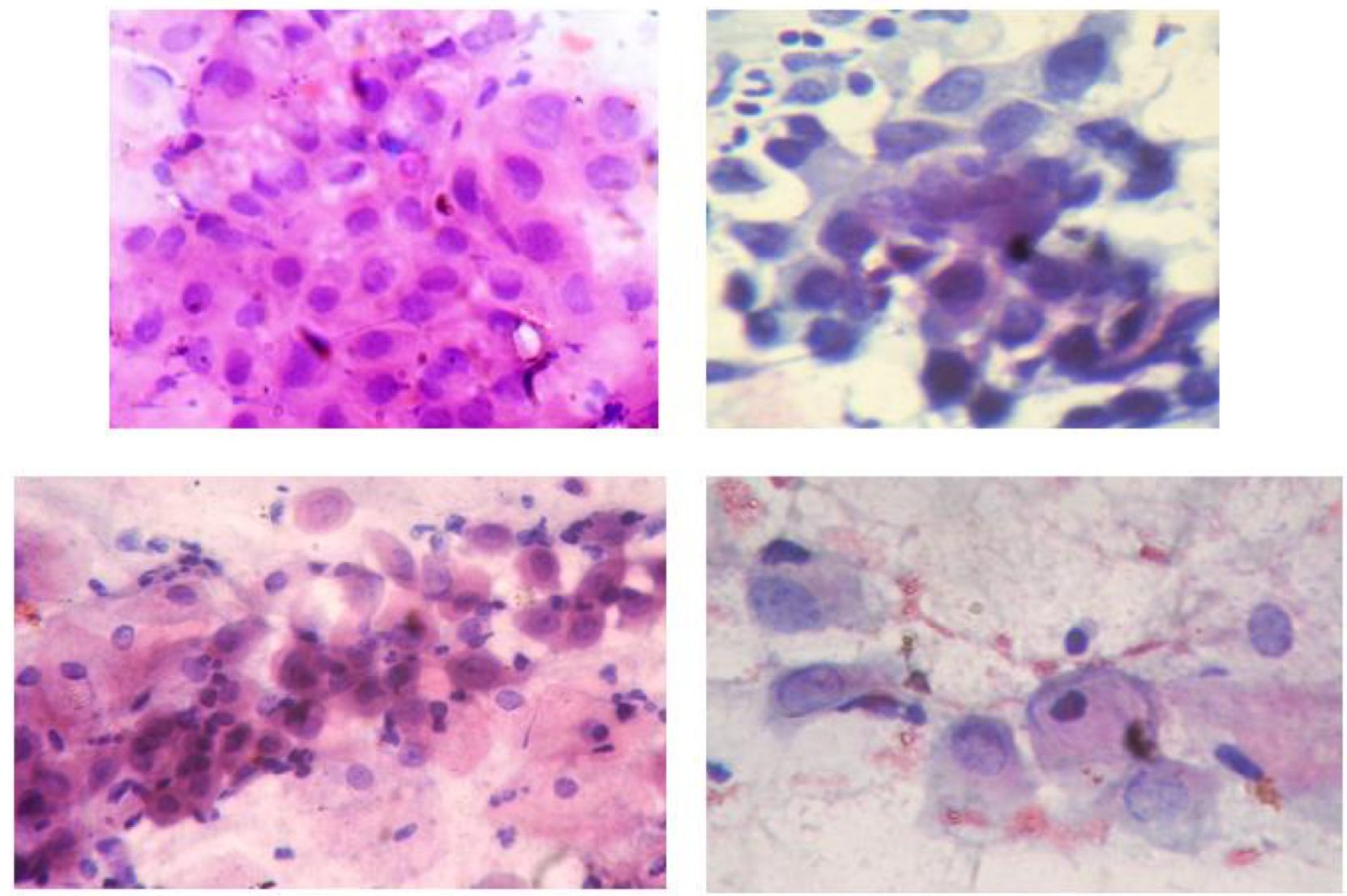

Figure 1: (A-D) : Low grade squamous intraepithelial lesion (A: Conventional Pap Stain, $\mathrm{x}$ 400) showing nuclear enlargement with anisonucleosis, irregular nuclear membrane and clumped to hyperchromatic nuclei; High grade squamous intraepithlial lesion (HSIL) (B: Conventional Pap Stain, x400) showing dysplastic cells arranged in syncytial cluster with anisonucleosis, high NC ratio, irregular nuclear membrane and hyperchromatic nuclei; atypical squamous cell of uncertain significance (ASCUS) (C: Conventional Pap Stain, x400) showing enlarged nuclei with minimal nuclear hyperchromasia and irregularity in nuclear membrane; Koilocyte (D : Conventional Pap Stain, x400) showing cytopathic effect of HPV with perInuclear clearing, peripheral condensation of cytoplasm and crumpled hyperchromatic nuclei.

\section{RESULTS}

The age distribution of 100 HIV-positive cases ranged from 19-51 years (mean: 33.70 years), while the age range of 75 HIV-negative cases varied between 22-52 years (mean: 32.29 years). The age group distribution among the two groups has been compared in table 1 which was not significant $(\mathrm{p}$ value 0.286 ).

There were total 41 cases of HR-HPV 16/18 positive cases in our study. Among them, 35 cases were HIV positive and 6 cases were HIV negative women. Thus, $35 \%$ of the HIV-positive women were found MJSBH Vol 16 Issue 2 July-Dec 2017 to be positive for HR-HPV $16 / 18$ whereas only $8 \%$ of HIV-negative women were positive for HR-HPV 16/18 [Table 2]. The result was found to be significant statistically ( $p$ value $<0.05$ ).

The spectrum of cervical precancerous lesions found on Pap smears comprised of low-grade squamous intraepithelial lesions (LSIL), high-grade squamous intraepithelial lesions (HSIL) and atypical squamous cells of uncertain significance (ASCUS) [Figure 1]. The distribution of the classified lesions varied among the two groups. LSIL, HSIL and ASCUS in HIV-positives were $16(16 \%), 3(3 \%)$ and $2(2 \%)$ 


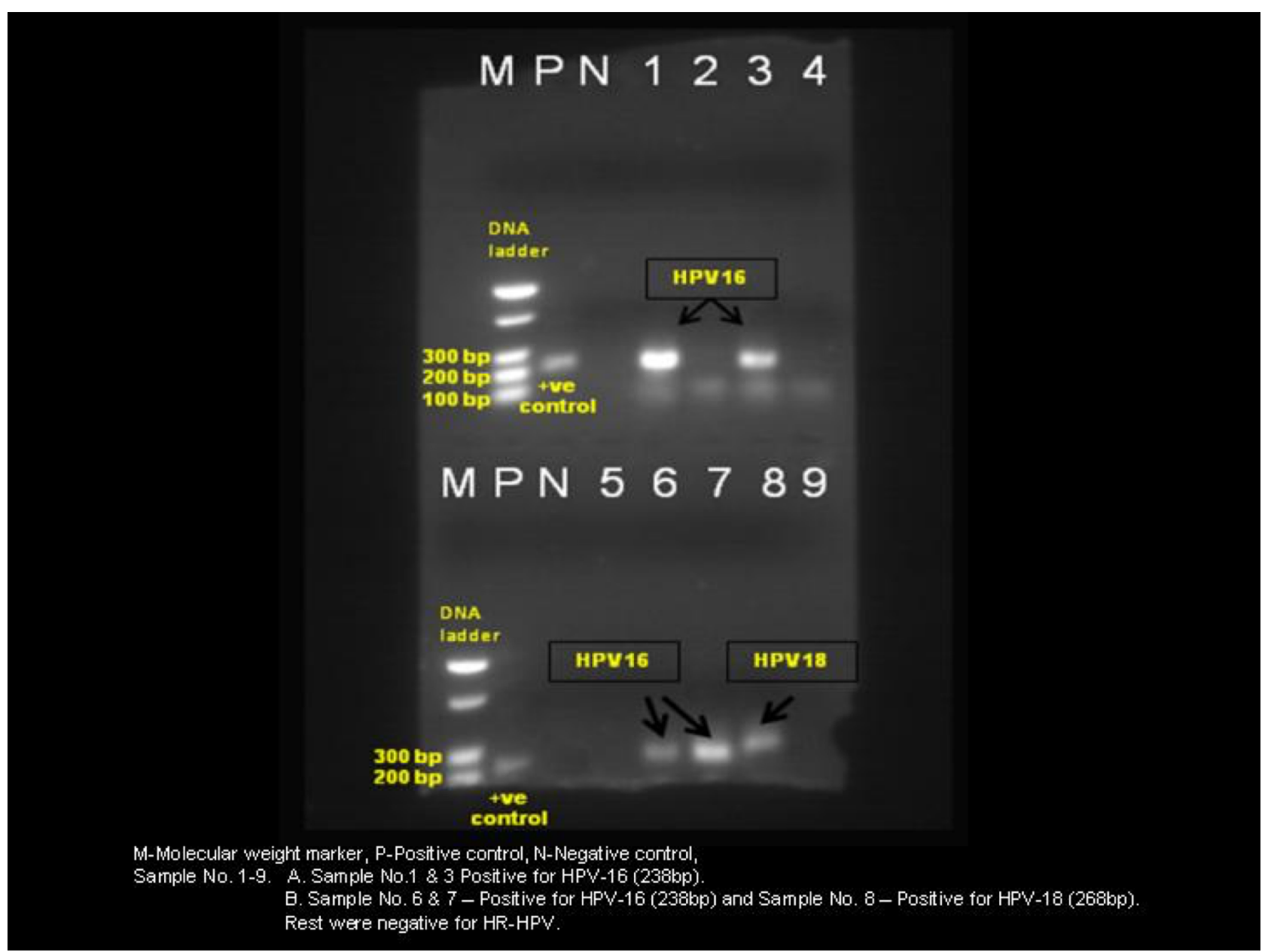

Figure 2: Figure 2 : HR-HPV Electrophoresis - Sample No. 1-9. A. Sample No.1 \& 3 was positive for HPV-16 (238bp). B. Sample No. 6 \& 7 were positive for HPV-16 (238bp) while sample No. 8 was positive for HPV-18 (268bp). Rest were negative for HR-HPV.

respectively while in HIV-negatives there were 4 $(5 \%), 2(2.5 \%)$ and $1(1.3 \%)$ cases, respectively [Table 3]. Thus, the prevalence of all the varied lesions was more on the HIV positive females than in HIV negative females. LSIL was found to be statistically significant in the HIV positive females with $\mathrm{p}$ value of 0.011 . Although the prevalence of HSIL and ASCUS lesions were more on the HIV positive cases than in HIV negative ones, the $\mathrm{p}$ values were 0.65 and 0.56 respectively and not significant statistically.

\section{DISCUSSION}

Cervical carcinoma is the second most common carcinoma among females in developing countries with more than 0.12 million cases being diagnosed annually ${ }^{4}$. Among various risk factors of cervical carcinoma, infection with HPV is one of the major preventable one ${ }^{1}$. HPV infection is a known cause of cervical cancer and about $70 \%$ of cervical cancer cases worldwide have been attributed to HPV 16 and 18 infections. ${ }^{4}$ Although the incidence of cervical carcinoma has been showing declining trends in developed countries, it is still a major burden in developing countries. ${ }^{5}$

Cervical smear cytology introduced by George Papanicolaou in early 1950s is being widely used as a screening tool for the early detection of cervical cancer and its precancerous lesions. Presently, cervical cancer screening guidelines also recommends using HPV detection, along with Pap 
smear6. However, cervical cancer and its precancerous lesions are still being diagnosed very late in most of the developing countries. Thus, early detection of HPV infection can be used as an effective screening mechanism for the prevention of cervical cancer, especially among HIV positive women in the developing regions of the world.

The burden of cervical cancer is also concurrent with the burden of HIV positivity. The immune suppression in HIV is known to facilitate the abnormal cervical cytology in women with HPV infection and increase the risk of cervical cancer. ${ }^{2,3}$ Thus to effectively control cervical cancer, it is necessary to screen for HPV infection and abnormal cytology, especially among HIV positive females and treat them appropriately.

The present study showed the prevalence of HRHPV (16/18) in HIV-positive women to be $35 \%$ and $8 \%$ in the HIV-negative women which was statistically significant [Table 2]. This result was comparable to various other studies which also showed similar association ${ }^{7-10}$. At the same time, it is worth noting that $80 \%$ cervical cancer cases have HR-HPV (16/18) infection 4 . The higher prevalence of HR-HPV 16/18 in immune suppressed status may thus correlate with development of cervical carcinoma especially among HIV positive women.

The analysis of the abnormal cytology in the HIV positive and negative women showed $16 \%$ and $8 \%$ prevalence of LSIL, $3 \%$ and $2.5 \%$ prevalence of HSIL and $2 \%$ and $1.3 \%$ prevalence of ASCUS in the two groups respectively [Table 3]. The prevalence of LSIL in HIV-positive women in the study (16\%) was significant statistically ( $p$ value 0.011 ). This prevalence is higher than the ones noted by Bagga et . al. (5.9\%) and Carlucci et al (8\%) respectively. ${ }^{11,12}$ The prevalence of LSIL in HIV negative women in the present study was $8 \%$. Froberg et. al. and Wright et. al. had reported the prevalence of LSIL in HIV negative to be $2.21 \%$ and $3.46 \%$ respectively. 13,14
Thus, there has been consistently higher rate of LSIL among HIV positive women than in HIV negative women substantiating the fact that immune suppression is correlated with abnormal cervical cytology.

Although HSIL and ASCUS lesions were also found to be higher among the HIV positive ones than in HIV negative ones, this finding was not statistically significant. Bagga et. al. and Carlucci et. al. too had noted HSIL $(2.2 \%$ and $6.2 \%$ respectively) and ASCUS lesions (1.5\% and $1.8 \%$ respectively) in their studies which was comparable to the present study. ${ }^{11,12}$ The prevalence of HSIL and ASCUS in HIV negative women $(2.7 \%$ each) in the current study was also akin to those reported by Wright et. al. and Gonzalez et. al. ${ }^{14,15}$ The significance of these findings needs to be substantiated with larger, multicentric studies.

In light of the association of HIV infection and HPV infection and abnormal cervical cytology, it has been recommended that contesting HIV-infected women with both cervical cytology and HPV Pap smear would be more appropriate for early detection of cervical cancer.8,16 Thus, early and appropriate treatment of HIV is very important for effective control of HPV infection and cervical carcinoma among HIV positive women.

\section{CONCLUSIONS}

A case control study was undertaken to study the prevalence of HPV infection and abnormal cervical cytology among HIV positive and negative women. The study showed significant association of HPV infection and abnormal cervical cytology among HIV positive females. Hence the study concludes that screening tests for cervical cancer should include both HPV detection and cervical cytology, especially in HIV positive women. Overall, prevention of cervical cancer in HIV positive women should be directed towards early detection of 
HPV and cervical cytological abnormalities schedule, which severely hampered subsequent followed by a prompt and appropriate treatment. biopsy confirmation.

The study is a single centric study with limited The above findings should be further corroborated number of subjects. The present study was limited with multi-institutional, adequately powered study by the poor patient compliance to regular follow-up to derive definite conclusions.

\section{REFERENCES}

1. Sun XW, Kuhn L, Ellerbrock TV, Chiasson MA, Bush TJ, Wright TC. Human Papillomavirus Infection in Women Infected with the Human Immunodeficiency Virus. NEJM 1997;337:1343-9.

DOI: https://doi.org/10.1056/NEJM199711063371903

2. Heard I, Tassie J-M, Schmitz V, Mandelbrot L, Michel D, Orth G. Increased risk of cervical disease among human immunodeficiency virus infected women with severe immunosuppression and human papillomavirus load. Obstet Gynecol 2000;96:403-9.

DOI: https://doi.org/10.1097/00006250-200009000-00016

3. Kanowitz S, Miller SB, Stone J, Hanson E. The Natural History of Human Papillomavirus Infection as Measured by Repeated DNA testing in Adolescent and Young Women. J Pediatr 1998;132:277-84.

DOI: https://doi.org/10.1016/S0022-3476(98)70445-7

4. Bruni L, Barrionuevo-Rosas L, Albero G, Aldea M, Serrano B, Valencia S, et al. ICO information centre on HPV and cancer (HPV information centre) Human Papilloma virus and related diseases in India. Summary Report 2015-03-20.

5. Asthana S, Chauhan S, Labani S. Breast and cervical cancer risk in India: an update. Indian J Public Health 2014;58(1):5-10

DOI: https://doi.org/10.4103/0019-557X.128150

6. Tao X, Griffith CC, Zhou X, Wang Z, Yan Y, Li Z, et al. History of high-risk HPV and Pap test results in a large cohort of patients with invasive cervical carcinoma: Experience from the largest women's hospital in China. Cancer Cytopathol $2015 \mathrm{Jul} ; 123(7): 421-7$

DOI: https://doi.org/10.1002/cncy.21545

7. Sarkar K, Pal R, Bal B, Saha B, Bhattacharya S, Sengupta S, et. al. Oncogenic HPV among HIV infected female population in West Bengal, India. BMC Infect Dis 2011;11:72.

DOI: https://doi.org/10.1186/1471-2334-11-72

8. Peedicayil A, Thiyagarajan K, Gnanamony M, Pulimood SA, Jeyaseelan V, Kannangai R, et. al. Prevalence and risk factors for human papillomavirus and cervical intraepithelial neoplasia among HIV positive women at a tertiary level hospital in India. J Low Genit Tract Dis 2009;13:159-64.

DOI: https://doi.org/10.1097/LGT.0b013e31818fb40d 
9. Zarcone R, Cardone G, Bellini P, Carfora E, Fortuna G, Raucci F, et. al. Incidence of HPV and CIN in HIV positive women. Minerva Ginecol 1995;47:477-80.

10. Joshi SN, Gopalkrishna V, Kumar BK, Dutta S, Nyaynirgune P, Thakar M, et. al. Cervical squamous intra-epithelial changes and human papillomavirus infection in women infected with human immunodeficiency virus in Pune, India. J Med Virol 2005;76:470-5.

DOI: https://doi.org/10.1002/jmv.20385

11. Bagga R, Wanchu A, Rajwanshi A, Gupta K, Prasad G, Gopalan S, et. al. Papanicolaou smear abnormalities in HIV-infected women in north India. Asia-Pacific Journal of Clinical Oncology 2005;1:77-80.

DOI: https://doi.org/10.1111/j.1743-7563.2005.00014.x

12. Carlucci M, Cimmino A, Fiore MG. The Pap test in HIV-positive women. Pathologica 2001;93:651-3.

13. Froberg M, Johansson B, Hjerpe A, Andersson S. Human papillomavirus 'reflex' testing as a screening method in cases of minor cytological abnormalities. Br J of Cancer 2008;99:563-8.

DOI: https://doi.org/10.1038/sj.bjc.6604504

14. Wright TC Jr, Denny L, Kuhn L, Pollack A, Lorincz A. HPV DNA Testing of self-collected Vaginal Samples Compared with cytologic screening to detect cervical cancer. JAMA 2000;283:81-6.

DOI: https://doi.org/10.1001/jama.283.1.81

15. González C, Canals J, Ortiz M, Mu-oz L, Torres M, García-Saiz A, et. al. Prevalence and determinants of high-risk human papillomavirus (HPV) infection and cervical cytological abnormalities in imprisoned women. Epidemiol Infect 2008;136:215-21.

DOI: https://doi.org/10.1017/S0950268807008382

16. Thaxton L, Waxman AG. Cervical cancer prevention: immunization and screening 2015. Med Clin North Am 2015;99(3):469-77.

DOI: https://doi.org/10.1016/j.mcna.2015.01.003 